\title{
Nordiques
}

36 | 2018

Réformer l'éducation en Europe du Nord

\section{La dimension temporelle de l'égalité : les réformes scolaires en Suède (1946-2000)}

\section{Tomas Wedin}

\section{(2) OpenEdition \\ 1 Journals}

Édition électronique

URL : http://journals.openedition.org/nordiques/529

DOI : $10.4000 /$ nordiques.529

ISSN : 2777-8479

Éditeur :

Association Norden, Bibliothèque de Caen la mer

\section{Édition imprimée}

Date de publication : 1 novembre 2018

Pagination : 99-114

ISBN : 9791095914020

ISSN : 1761-7677

Référence électronique

Tomas Wedin, «La dimension temporelle de l'égalité : les réformes scolaires en Suède (1946-2000)», Nordiques [En ligne], 36 | 2018, mis en ligne le 31 octobre 2020, consulté le 13 mars 2021. URL : http:// journals.openedition.org/nordiques/529; DOI : https://doi.org/10.4000/nordiques.529 


\section{La dimension temporelle de l'égalité : les réformes scolaires en Suède (1946-2000)}

Tomas Wedin*

\section{RÉSUMÉ}

Selon l'interprétation dominante dans l'historiographie éducative, le système éducatif suédois a connu une rupture idéologique vers 1990. À rebours de cette tradition, cet article met en évidence une certaine continuité entre la pensée des années 1970, soit la phase culminante de l'ordre égalitaire de l'après-guerre, et les réformes des années 1990, souvent considérées comme la manifestation d'une rupture avec la doctrine éducative préexistante. Afin de faire ressortir les lignes de continuité, j'analyse ici comment la manière dont l'idéal d'égalité a été mobilisé dans le contexte des politiques éducatives a influencé la référence au passé, au présent et au futur en tant qu'horizons de l'action collective. Je soutiens que le présent est devenu de plus en plus l'horizon de référence au détriment autant du passé que du futur, et que la radicalisation de l'aspiration à l'égalité a renforcé cette transformation. En concluant, je soutiens que les efforts dans le sens de la démocratisation du système éducatif ont paradoxalement sapé sa légitimité fondée sur un ordre collectif.

\section{ABSTRACT}

According to the dominant strand of educational historiography, the Swedish educational system underwent an ideological break around 1990. In contrast, this article accentuates a certain continuity between the educational thought of the 1970s, the culminating phase of the egalitarian order of the post-war period, and the reforms of the 1990s, often considered as the manifestation of a break with the pre-existing educational doctrine. In order to evince these strands of continuity, I analyse how the ways in which the ideal of equality has been mobilized in educational policies ties in with different ways of relating to the past, present and future as temporal horizons for collective action. I argue that the present successively has become the horizon of reference at the expense of both the past and the future, and that the radicalization of the aspiration for creating a more equal educational system spurred this shift. To conclude, I argue that the efforts to democratise the educational system have paradoxically sapped its own legitimacy, which hinges on the existence of a collective order.

\footnotetext{
* Doctorant en histoire des idées à l'université de Göteborg.
} 
Le processus de démocratisation de la société a changé, à bien des égards, notre vision des objectifs de l'école, de ses problèmes d'organisation, de l'étendue de son activité, du contenu de l'enseignement et des méthodes éducatives. Cette modification de notre conception de l'école et de ses fonctions n'a pas encore su imprégner la vie scolaire entièrement, pour des raisons évidentes. Le temps semble maintenant être venu de donner à l'école les moyens dont elle a jusqu'ici manqué ${ }^{1}$.

Entre l'après-guerre et la seconde moitié des années 1980, les stratégies de réforme qui ont dominé la politique suédoise dans le domaine de l'éducation se sont caractérisées - pour reprendre les mots d'une enquête influente, la Maktutredning (Audit du pouvoir) - par une " tradition de démocratie centrée sur la société " (en samhällscentrerad demokratitradition), dans laquelle l'idéal d'égalité occupe une place dominante. En revanche, les réformes réalisées en Suède depuis la fin des années 1980 ont souvent été interprétées comme une rupture avec ce modèle, la liberté individuelle se substituant progressivement à l'idéal d'égalité ; elles témoigneraient ainsi de la métamorphose d'une idéologie sociale teintée de collectivisme en individualisme ${ }^{2}$. Après avoir été l'un des systèmes éducatifs les plus centralisés, le système éducatif suédois serait donc devenu l'un des plus décentralisés et des plus libéralisés au monde, ce qu'atteste l'apparition d'acteurs privés sur le marché éducatif, qui marque le début des années $1990^{3}$. L’introduction d'un système de " chèques-éducation " (vouchers), c'est-à-dire un mode de financement public indirect de l'enseignement privé, au début des années 1990, en est à ce titre une bonne illustration. D'après ce système, chaque municipalité choisit d'investir une somme donnée pour chaque élève (selon le niveau scolaire ainsi que ses choix de spécialisation au lycée), qui le suivra tout au long de son parcours scolaire.

À rebours de cette interprétation dominante qui met en avant une rupture idéologique, cet article s'efforcera de mettre en évidence une certaine continuité entre la pensée des années 1970, soit la phase culminante de l'ordre égalitaire de l'après-guerre, et les réformes des années 1990, souvent considérées comme la manifestation d'une rupture avec la doctrine éducative préexistante. La dynamique

1 SOU $1946: 27$, p. 1 .

2 Tomas Englund (éd.), Utbildningspolitiskt systemskifte ?, Stockholm, HLS, 1996 ; Tomas Englund et Anne Quennerstedt, Vadå likvärdighet, Göteborg, Daidalos, 2008 ; Tomas Englund, " Education as a citizenship right ", Journal of Curriculum Studies, n² 26, juillet 1994, p. 383-399 ; Ylva Boman, Utbildningspolitik i det andra moderna. Om skolans normativa villkor, Örebro, Örebro universitet, 2002 ; Mattias Börjesson, Från likvärdighet till marknad. En studie av offentligt och privat inflytande över skolans styrning i svensk utbildningspolitik 1969-1999, Örebro, Örebro universitet, 2016 ; Sara Carlbaum, Blir du anställningsbar lillela vän? Diskursiva konstruktioner av framtida medborgare i gymnasiereformer 1971-2011, Umeå, Umeå universitet, 2012.

3 Andreas Fejes et Magnus Dahlstedt, Skolan, marknaden och framtiden, Lund, Studentlitteratur, 2018. 
sous-tendant les évolutions de l'idée d'égalité de 1946 à 2000 sera appréhendée comme un facteur clé pour mieux comprendre ce changement d'orientation. Plus précisément, je vais analyser comment la manière dont l'idéal d'égalité a été mobilisé dans le contexte des politiques éducatives a influencé la référence au passé, au présent et au futur en tant qu'horizons de l'action collective. Il me semble en effet que l'évolution de la compréhension dominante de l'égalité est intimement liée aux différentes façons d'appréhender le rapport au temps. Je soutiendrai que le présent est devenu de plus en plus l'horizon de référence au détriment autant du passé que du futur, et que la radicalisation de l'aspiration à l'égalité a influencé cette transformation. De plus, je montrerai comment les efforts dans le sens de la démocratisation du système éducatif ont paradoxalement sapé sa légitimité fondée sur un ordre collectif.

Cette manière « politico-historique » d'aborder le système éducatif est inspirée des travaux de François Hartog sur les régimes d'historicitét. Dans un livre publié en 2003, Hartog analyse l'histoire de l'Occident à travers la manière dont elle s'est déployée dans le temps, c'est-à-dire la manière dont elle se rapporte aux trois dimensions temporelles. D'après Hartog, depuis l'après-guerre, le régime d'historicité orienté vers le futur, dominant depuis la Révolution française, a été remplacé par un régime présentiste ${ }^{5}$. En reprenant le point de vue d'Hartog, j'analyserai comment les réformes éducatives suédoises témoignent d'une transmutation successive de l'articulation de l'idéal d'égalité avec les trois dimensions temporelles.

Mon corpus comprend des documents officiels portant sur différentes réformes. Je m'appuierai principalement sur des projets de loi (propositioner) ainsi que sur des rapports de commissions d'enquête (Statens offentliga utredningar, $\mathrm{SOU})^{6}$. Ces derniers sont le reflet d'une ancienne tradition de régulation étatique suédoise, visant à enquêter sur un problème donné avant de formuler une proposition législative. Leur but est de circonscrire différents aspects de la question concernée, d'analyser les problèmes mis en évidence par le ministre qui les a

4 François Hartog, Régime d'historicité, Paris, Seuil, 2012. Lapproche d'Hartog est de son côté profondément inspirée de la pensée de Reinhard Koselleck, notamment le couple conceptuel Erfahrungsraum et Erwartungshorizont, voir : Reinhard Koselleck, Vergangene Zukunft. Zur Semantik geschichtlicher Zeiten, Francfort-sur-le-Main, Suhrkamp, 1992.

5 Pour une analyse spécifiquement vouée au concept de Hartog d'un régime présentiste pour analyser le système éducatif, voir : Tomas Wedin, "In Praise of the Present : the pupil at centre in Swedish educational politics in the post-war period ", History of Education, ${ }^{\circ}$ 46, novembre 2017, p. 768-787.

6 J'utiliserai aussi le curriculum pour la grundskola de 1980. Pour des examens plus approfondis, renforçant la dynamique esquissée ici, voir : Piero Colla, L’héritage impossible. Conscience historique et technologies de lidentité dans la réforme éducative en Suède 1946-1980, Paris, EHESS, 2017 ; Tomas Wedin, "In Praise... ", op. cit. ; Tomas Wedin, "The paradox of democratic equality : on the modified teacher role in post-war Sweden ", Confero, n 5, décembre 2017, p. 383-399. 
mandatés, ainsi que, le plus souvent, de recommander des mesures pour les résoudre. Ainsi, bien qu'ils ne correspondent pas nécessairement aux décisions prises par la suite, ces documents offrent un arrière-plan intéressant pour saisir des tendances des débats politiques.

L'analyse se déploiera en trois temps. Dans un premier temps, je me concentrerai sur l'influente enquête de 1946 (1946-års skolkommissions betänkande), puis sur les changements dans les années 1970 ; enfin, sur les transformations dans les années 1990. La problématique centrale - le rapport entre l'idéal d'égalité et les trois dimensions temporelles - sera examinée sur fond d'une analyse des transformations de la relation entre trois pôles : l'élève, le professeur et les contenus disciplinaires.

\section{DireCTIVES GÉNÉRALES POUR UNE DÉMOCRATISATION DU SYSTÈME ÉDUCATIF SUÉDOIS ${ }^{7}$}

Même si une enquête globale sur l'état et les perspectives de réforme du système éducatif (4000 pages réparties en vingt rapports) était en cours de préparation depuis 1940 , ce n'est qu'au sortir de la guerre que le gouvernement socialdémocrate décida d'engager une commission d'enquête, sur la base d'un nouveau mandat. Cette fois-ci, elle devait viser une modernisation complète du système scolaire. Le gouvernement de l'époque justifia l'initiative par le fait que d'autres réformes majeures étaient sur le point d'être lancées. Comme ces réformes allaient s'avérer onéreuses et qu'il fallait les justifier, une nouvelle commission fut créée ${ }^{8}$.

L'augmentation rapide du taux de natalité après la paix poussa le gouvernement à adopter une approche plus exhaustive que celle de l'enquête de 1940 ; les enjeux de réforme y incluent la formation des enseignants, les bâtiments scolaires et le reste du complexe éducatif. L'école, déclarait le comité dès la première page, devrait dès maintenant viser une " démocratisation du système scolaire suédois »" Ce nouvel élan dans la politique éducative était une réaction évidente à l'expérience des régimes totalitaires et de la guerre ${ }^{10}$.

L'école démocratique devrait, d'après les membres de la commission, devenir " un environnement libre pour le développement des enfants ", l'individualité et les prédispositions personnelles des élèves en étant le point de départ ${ }^{11}$. Cette

7 SOU $1948: 27$, p. 1.

8 SOU 1948 : 27, p. x. Pour une analyse du rapport entre les deux enquêtes, voir : Gunnar Richardson, Drömmen om en ny skola. Idéer och realiteter i svensk skolpolitik 1945-1950, Stockholm, Liber/Allmänna Förlag, 1983.

9 SOU $1948: 27$, p. 1.

10 Voir, par ex. : Johan Östling, Nazismens sensmoral: svenska erfarenheter $i$ andra världskrigets efterdyning, Lund, Lunds universitet, 2008 ; Gunnar Richardsson, Drömmen, op. cit.

11 SOU $1948: 27$, p. 3, $22 \mathrm{f}$. 
focalisation sur l'élève allait de pair avec un idéal d'une école qui perpétue une culture générale. D'après cette vision, ce n'est que lorsque l'apprentissage est libre, que l'élève peut s'immerger dans la culture ${ }^{12}$. C'est donc seulement par le moyen d'une éducation libre que l'école peut contribuer au développement de la communauté démocratique, qui requiert des citoyens éclairés. Ainsi, la formation de citoyens libres, le respect de l'héritage culturel et l'apprentissage libre étaient considérés comme indissociables.

Le lien complexe entre ces trois dimensions est encore renforcé par l'importance attribuée à l'école en tant qu'espace visant à affiner le goût des élèves. Afin d'éviter la diffusion endémique du divertissement de mauvaise qualité - qu'il s'agisse de films, de musique ou de littérature -, l'école était censée transmettre une éducation esthétique aux élèves pour qu'ils puissent "s'approprier les trésors de la vie culturelle et vivre de ce fait une vie plus riche ${ }^{13}$. En guise d'exemple, il était considéré comme essentiel que les enfants « à un stade précoce, apprennent à apprécier les valeurs esthétiques de la poésie et, avec le temps, sachent préférer la bonne littérature à la mauvaise $»^{14}$. Cependant, l'ambition ne consiste pas à donner à chaque élève un bagage de connaissance uniforme. Il semble plus important de fournir aux enfants, conformément à l'intérêt de chacun, la possibilité d'approfondir leurs connaissances dans des domaines spécifiques ${ }^{15}$. Bien qu'offrir un choix plus large à chaque élève du contenu étudié était recommandé, cette liberté s'exerce toujours dans un cadre précis : la transmission du patrimoine culturel. Les élèves devraient être éduqués à devenir citoyens et, en tant que tels, des égaux, à travers un héritage commun. Ainsi l'école était-elle censée introduire les élèves dans le monde tel qu'il est considéré par les adultes, afin de préparer ces citoyens en devenir.

L'idéal d'égalité sur lequel cette conception repose implique la participation collective à un tel processus de transmission. Ce qui n'exclut pas l'attention vis-àvis du monde environnant et actuel : ainsi, les auteurs du rapport déploraient-ils que l'enseignement de la littérature et de l'histoire soit souvent abstrait et déconnecté de la réalité des élèves ${ }^{16}$. C'est en réaction contre une telle tendance que les enquêteurs proposaient de faire de l'" éducation sociale " (Sambällskunskap) une nouvelle matière obligatoire ${ }^{17}$.

\footnotetext{
12 Ibid., p. 3.

13 Ibid., p. 7.

14 Ibid., p. 31.

15 Ibid.

16 Comme l'a méticuleusement montré Piero Colla, L’héritage impossible, op. cit.

17 SOU $1948: 27$, p. 30. C'est à la lumière de ces changements que l'introduction de l'éducation civique (Sambällskunskap) comme nouvelle matière doit être comprise.
} 
D'une manière symptomatique, le compte rendu prend alors un tournant argumentatif, en intégrant la critique d'une éducation trop détachée de l'actualité dans un cadre plus vaste :

Ce serait néanmoins fatal si l'école considérait la transmission des connaissances d'un point de vue exclusivement pratique. La transmission du savoir devrait finalement viser le développement de la personnalité, la conscience culturelle, la compréhension de la place de l'humanité dans l'univers, l'humilité devant la mystique de l'existence et la recherche de sa place dans le monde. ${ }^{18}$

Comme on le constate, le cadre dans lequel chaque élève doit s'inscrire s'étend clairement au-delà de la sphère restreinte de ses intérêts privés. L'allusion à la mystique et au sens de la vie ainsi que la toile de fond commune à travers laquelle l'enfant est supposé s'élever, témoignent d'une ambition éducative qui permet à l'élève de s'élever au-delà de ses intérêts immédiats ${ }^{19}$.

Même si ces rapports réclamaient une transformation importante du système éducatif, ils insistaient sur la centralité de la transmission de savoirs dans l'enseignement. Une preuve en est notamment l'importance accordée au développement d'un contenu normatif qui contribue à la formation des élèves. Mais comme cela sera démontré plus loin, cette référence sera par la suite de plus en plus contestée.

\section{VERS UN APPROFONDISSEMENT DE LA DÉMOCRATISATION DU SYSTÈME ÉDUCATIF}

Pendant les années 1970, la compréhension dominante de la relation élèveenseignant ainsi que l'image officielle de l'école connaissent une métamorphose. La contestation sociale menée par différents mouvements, à partir de la fin des années 1960, visait le système éducatif en particulier ${ }^{20}$. Ce dernier était le lieu par excellence où règnent des relations inégales, la génération des aînés étant encore chargée de transmettre aux plus jeunes le savoir dont elle est dépositaire; comme dans le reste du monde occidental, l'école devint un objet central de critique. Olof Palme, alors ministre de l'Éducation (1967-1969), et bientôt premier ministre, formula la réflexion suivante : si un "approfondissement de la démocratie dans

18 SOU $1948: 27$, p. 30 .

19 Rappelant ainsi les origines dans l'idéalisme allemand du "folkbildning " tellement central dans le mouvement ouvrier en Suède pendant la première moitié du XX siècle. Pour ne mentionner qu'un exemple très représentatif de ce courant dans le mouvement, voir les réflexions de l'ex-ministre et initiateur de la Fédération ouvrière d'éducation populaire (ABF) Richard Sandler, dans Richard Sandler, Mångfald eller enfald. Ta loch artiklar till Arbetarnas Bildningsförbunds 25-årshögtid, Stockholm, Tiden, 1937.

20 Ainsi, quand le ministre de l'Éducation Olof Palme, dans la deuxième chambre du Parlement, déclara que l'école devait être considérée comme «la clé pour le renversement de la société de classes », il prononça une critique beaucoup plus générale de l'époque. Voir : "Skolan är och förblir en nyckel till att avskaffa klassamhället ", Andra kammarens protokoll, 41, 1968, p. 72. 
notre société » est ce que nous voulons, alors les jeunes doivent avoir « la possibilité d'appliquer des méthodes démocratiques dès l'école $»^{21}$. L'école fut, en outre, critiquée pour son asservissement aux demandes du marché du travail. Elle était considérée comme responsable de la reproduction de la société de classes ${ }^{22}$. C'est dans ce contexte historique que les documents analysés ci-dessous furent élaborés.

En ce qui concerne l'organisation scolaire, la citation suivante, extraite de l'enquête publique intitulée L'individu et l'école, révèle le climat politique régnant à l'époque et comment les enquêteurs interprétaient leur tâche par rapport aux réformes éducatives des décennies précédentes :

On pourrait maintenant supposer que les réformes les plus ambitieuses concernant les contours du système éducatif préuniversitaire seront accomplies dans un avenir prévisible. Pour le système scolaire, la tâche est maintenant d'essayer de trouver des moyens encore meilleurs pour que tous les enfants et adolescents soient stimulés par leurs devoirs et que l'enseignement leur donne du sens. ${ }^{23}$

L'un des changements qui saute aux yeux est la disparition totale, au cours des années 1970, des références au patrimoine culturel ou au goût de la culture : autant de témoignages d'une croyance persistante en l'importance de confronter les élèves à un contenu partagé/commun ${ }^{24}$. C'est l'indice d'un changement symbolique non négligeable : une rupture avec l'idée que les connaissances se trouvent à l'extérieur de l'individu et le transcenden $\mathrm{t}^{25}$. Cela révèle aussi une transition vers une relativisation de l'importance du contenu de l'enseignement, et une confiance augmentée dans le rôle des formes dans l'enseignement afin de faire progresser la démocratisation de l'école ${ }^{26}$.

Les élèves apparaissent désormais comme des sujets démocratiques. L'enquête sur le fonctionnement interne du système scolaire (Utredningen om skolans inre

21 Gunnar Richardson (éd.), Minnen och dokument. 9, Spjutspets mot framtiden, Uppsala, Föreningen för svensk undervisningshistoria, 1997, p. 44.

22 Ylva Boman, Utbildningspolitik i det andra moderna., op. cit. ; Tomas Englund, Läroplanens och skolkunskapens politiska dimension, Göteborg, Daidalos, 2005, p. 268-272 ; Gunnar Richardson, Svensk utbildningshistoria - skola och sambälle för och nu, Lund, Studentlitteratur, 2010, p. 14, p. 138-140 ; Ninni Wahlström, Om det förändrade ansvaret för skolan, Örebro, Örebro universitet, 2002, p. 53 ; Mattias Börjesson, Från likvärdighet till marknad, op. cit. ; Johanna Ringarp, Professionens problematik. Lärarkårens kommunalisering och välfärdsstatens förvandling, Stockholm, Makadam, 2011, p. 39f., p. 46.

23 SOU 1975 : 9, p. 11, p. 106.

24 Tomas Wedin, «In Praise... ", op. cit.

25 Cette tendance est clairement discernable également dans les débats autour du concept de la culture et la politique culturelle de l'État pendant les années 1960-1970. Voir : Anders Frenander, Kulturen som kulturpolitikens stora problem, Möklinta, Gidlunds, 2014.

26 Tomas Wedin, "The paradox... ", op. cit. 
arbete), présentée en 1974, en est une illustration ${ }^{27}$. Dans les directives du gouvernement social-démocrate, il est indiqué que :

Parmi les tâches de l'école, nous ne trouvons pas seulement la transmission des connaissances ; une mission tout aussi importante est de donner aux élèves la possibilité de se développer en tant que citoyen indépendant ayant un engagement personnel par rapport au monde environnant. Aucun élève ne peut être privé de ces opportunités parce qu'il connaît des difficultés dans le travail scolaire. L'école a, au contraire, une responsabilité particulière envers ces élèves, étant donné que le temps scolaire pourrait être une occasion unique, permettant de leur donner ces opportunités de développement personnel et social, que la société est tenue d'offrir à ses citoyens. ${ }^{28}$

Ainsi, le ministre de l'Éducation, dans la définition du mandat des enquêteurs, les chargea d'adapter l'enseignement afin d'améliorer la capacité de l'école à accueillir des enfants de milieux défavorisés ${ }^{29}$. De telles réformes, selon les enquêteurs, rendraient l'école plus juste dans le sens où elles diminueraient l'avantage que les enfants de classes aisées tendent à obtenir de l'école ${ }^{30}$. L'aspiration à créer une école plus démocratique et égalitaire, qui sous-tend cette enquête, se traduit notamment par l'injonction de veiller sur les élèves qui éprouvent les plus grandes difficultés à l'école.

Peu de temps après que les enquêteurs avaient présenté leurs conclusions, on retrouva la même idée - approfondir l'adaptation de l'enseignement aux expériences individuelles des élèves - dans une proposition de loi sur le fonctionnement interne de l'école. L'initiative venait du gouvernement social-démocrate de l'époque, dirigé par Olof Palme, avec Lena Hjelm-Wallen comme ministre de l'Éducation :

Le point de départ pour les propositions suivantes est que les municipalités et les unités scolaires de chaque municipalité devraient être autorisées à adapter l'enseignement aux besoins de différents élèves ou groupes d'élèves [...] L'école doit, dans une plus grande mesure qu'aujourd'hui, viser des formes de fonctionnement qui se rapprochent de la réalité des élèves. ${ }^{31}$

Quelle vision de l'enseignant se manifeste dans cette proposition ? Et comment ces glissements peuvent-ils être compris du point de vue qui est le nôtre, c'est-à-dire en analysant la manière dont la notion d'égalité se reflète sur les trois dimensions temporelles?

27 Utredningen om skolans inre arbete SOU 1974: 53 (SIA).

28 SOU $1974: 53$, p. 64.

29 SOU $1974: 53$, p. 64.

30 SOU $1974: 53$, p. $304 \mathrm{f}$.

31 Proposition $1975 / 76: 39$, p. 1 . 
Comme je l'ai souligné plus haut, la dimension véritablement subversive de ces changements est l'importance progressivement donnée aux formes pédagogiques aux dépens du contenu de l'enseignement. Cette tendance indique une vision radicalement nouvelle de l'équilibre entre le passé, le présent et le futur dans la politique éducative. En d'autres termes, il s'agit d'une nouvelle manière pour la société, à travers l'école en tant qu'institution fondatrice, de se représenter dans la ligne du temps, dans son projet pour les nouvelles générations. Le présent devient de plus en plus un horizon de référence exclusif : au détriment du passé, par la perte d'importance de l'assimilation d'un contenu ; et au détriment du futur, comme le montre l'accent porté sur la situation contingente de l'élève et l'injonction consistant à développer des pratiques démocratiques à l'école ${ }^{32}$. En effet, comme le prétendent les enquêteurs chargés d'élaborer le document "Considération sur la nouvelle formation de professeurs en 1974, SOU 1978 : 86 ", l'école devrait inciter les élèves à « la réalisation de soi, à des expériences du vivreensemble et du travail solidaire en communauté dans le temps présent $»^{33}$.

Il s'agit, en d'autres termes, d'une transformation de l'école, où sa fonction préparatoire, accompagner les élèves pour devenir des citoyens, est remplacée par l'idée selon laquelle l'école devrait être considérée comme une partie de la sphère publique. Cette transmutation indique aussi une nouvelle manière pour la société d'ordonner son rapport au temps. Comme nous l'avons vu, dans la grande enquête publique (SOU) de l'après-guerre, la société était envisagée dans son articulation avec le passé et le patrimoine commun. Lélève devait avoir connaissance du passé pour devenir un citoyen. En revanche, dans les années 1970, nous pouvons entrevoir comment un ordre temporel nouveau, mettant l'accent sur le présent, semble être entrelacé avec une désagrégation de la distinction entre l'école et la société environnante. L'école est de plus en plus perçue comme partie intégrante de la sphère publique ${ }^{34}$. Voilà comment le souhait d'intensifier la démocratisation à travers le rapprochement de l'école et de la société rencontre une nouvelle manière de s'orienter au regard du temps. L'aboutissement paradoxal de cette initiative est que la démocratisation de l'école a fini par faire des expériences personnelles et des intérêts des élèves, donc de ce qu'ils sont et font dans leur vie privée, une priorité pour l'enseignement.

32 Pour une analyse de la façon dont ces changements du système éducatif suédois peuvent être compris comme un glissement successif, pour parler comme François Hartog, d'un régime d'historicité futuriste à un régime en priorité présentiste, voir : Tomas Wedin, "In Praise », op. cit. Dans le quatrième tome de L'avènement de la démocratie, Marcel Gauchet évoque de manière plus générale la montée du présent comme le principal horizon d'orientation des démocraties occidentales, depuis 1970 : Marcel Gauchet, L'avènement de la démocratie IV : Le nouveau monde, Paris, Gallimard, 2017.

33 Betänkande av 1974-års lärarutbildning: Lärare för skola i utveckling SOU 1978:86 (LUT 74), 78.

34 Voir par exemple SOU 1974 : 84. 
À côté de cette entreprise d'adaptation de l'école aux goûts et personnalités spécifiques des élèves, nous retrouvons aussi les objectifs et directives de l'école élémentaire et du collège (grundskola) dès les premières pages du nouveau curriculum de 1980 : « l'École a l'obligation de donner aux élèves une plus grande responsabilité et une plus grande influence en fonction de leur âge et de leur maturité $"^{35}$.

Cette percée ultra-démocratique implique un changement au niveau de l'idée même d'égalité. Auparavant, il s'agissait de préparer les élèves à devenir des citoyens à travers l'école ; les transformations des années 1970 se fondent au contraire sur un idéal d'égalité devant se réaliser ici et maintenant. En essayant de faire avancer la démocratisation de l'école en la rendant plus égalitaire, les textes officiels témoignent d'une intention d'approcher l'individu là où il se trouve en renonçant à l'ambition de lui transmettre un contenu prédéterminé. En d'autres termes, c'est à la société de s'adapter à l'individu, et non l'inverse ${ }^{36}$. Je soutiens donc que l'importance croissante attribuée aux expériences et trajectoires privées des élèves, motivée en partie par un souci de rendre l'école plus égalitaire et démocratique, déporte l'attention vers le temps présent au détriment du passé ainsi que, dans une certaine mesure, du futur. L'enseignant devait désormais exercer de plus en plus un rôle de soutien de l'individu, non plus seulement un rôle de guide qui l'invitait à transmettre aux jeunes générations les valeurs de l'ancien monde ou des compétences pour une insertion ultérieure sur un marché du travail : "Éduquer pour la démocratie doit impliquer que l'élève soit stimulé à chercher et traiter des connaissances de manière autonome, et que la relation entre élève et enseignant prenne la forme d'une interaction démocratique. ${ }^{37}$

Cette évolution de la politique éducative coïncide avec des tendances plus générales en Occident pendant cette période ${ }^{38}$. Elle s'inscrit dans une critique sociale qui s'est développée à l'issue des Trente Glorieuses en Europe occidentale, aux États-Unis et au Japon. On le voit notamment à travers le courant antiautoritaire qui traversa de nombreuses sociétés. Ainsi, quand en 1976 le parti social-démocrate perd le pouvoir pour la première fois depuis 1932, la critique de

35 Mål och riktlinjer för grundskolan: Läroplan för grundskolan 1980 (Lgr 80), p. 15.

36 Ylva Boman, Utbildningspolitik $i$ det andra moderna, op. cit. ; Tomas Englund, Läroplanens, op. cit., p. 268-272 ; Gunnar Richardson, Svensk utbildningshistoria, op. cit., p. 14, p. 138-140 ; Ninni Wahlström, Om det förändrade, op. cit., p. 53 ; Mattias Börjesson, Från likvärdighet till marknad, op. cit.; Johanna Ringarp, Professionens problematik, op. cit., p. 39, p. 46.

37 SOU $1978: 86,78$.

38 Voir Marcel Gauchet, L'avènement de la démocratie, op. cit. 
la bureaucratie et l'incapacité à répondre à ce défi sont considérées comme une des causes principales de la défaite électorale ${ }^{39}$.

Néanmoins, et cela mérite d'être souligné, il ne faudrait pas considérer ces mouvements comme l'indice d'une rupture définitive avec les conceptions de l'éducation et de l'égalité mises en évidence dans les rapports précédents. Il est question d'un changement progressif, mais qui n'influence pas de manière fondamentale une certaine continuité de la démocratisation du système éducatif ${ }^{40}$. Comme on l'a vu plus haut, des tendances comparables sont clairement identifiables dès l'époque de l'après-guerre en 1946 . On peut le constater en particulier dans l'ambition de rapprocher l'école de la société, dans les idées d'inspiration progressiste d'un enseignement axé sur l'enfant et, finalement, dans la critique visant l'autorité à l'école ${ }^{41}$.

\section{UNE AUTORITÉ NÉGOCIÉE}

Les réformes éducatives des dernières décennies ont eu lieu dans un monde en pleine mutation. Des vagues de réfugiés - liées notamment à la guerre Iran-Irak pendant les années 1980 et aux guerres civiles en ex-Yougoslavie ou en Somalie - ont participé à modifier la structure de la société suédoise. Simultanément, la Suède a connu une crise économique profonde au début des années 1990. L'un des effets les plus spectaculaires des flux migratoires et de la crise économique fut l'entrée du parti populiste et xénophobe, Ny demokrati (La nouvelle démocratie), au Parlement suédois. Dans le secteur éducatif, l'utilisation toujours plus intensive de nouveaux moyens de communication a produit des attentes très importantes ${ }^{42}$. Pendant cette période, le système éducatif semble avoir achevé la transformation idéologique qui l'entraîne d'un modèle collectiviste vers un modèle de plus en plus centré sur l'individu.

Comme indiqué dans l'introduction, un exemple éloquent de ce changement vers une société nouvelle est la réforme introduisant le principe de la liberté de choix dans la sphère éducative, adoptée en 1992 sous un gouvernement de centre

39 Niklas Stenlås, «En kår i kläm - Läraryrket mellan professionella ideal och statliga reformideologier », Expertgruppen för studier i offenlig ekonomi 2009: 6, Department of Finance, p. 11.

40 Une idée qui est plus détaillée dans : Piero Colla, L’héritage impossible, op. cit. ; Tomas Wedin, "Tocqueville, Equality and Individualisation : On the Democratisation of Pre-University Education in Postwar Sweden », Lychnos, janvier 2019.

41 Ce rapprochement a été soigneusement analysé par Colla, voir : Piero Colla, L’héritage impossible, op. cit.

42 Pour une analyse autour de la façon dont la numérisation a été mobilisée comme un moyen de résoudre les problèmes de l'école, et comment cette tendance était symptomatique des nouvelles manières de se figurer le rôle du système éducatif, voir : Thomas Karlsohn, Teknik - Retorik - Kritik. Om ITbubblan och datoriseringen av den svenska skolan, Stockholm, Carlsson, 2009. 
droit. Trois ans auparavant, les sociaux-démocrates avaient déjà décentralisé la gestion des écoles. Dorénavant, les municipalités pouvaient elles-mêmes adapter l'offre de formation scolaire à leur gré, selon leurs besoins spécifiques, mais sous la surveillance de la nouvelle Direction nationale des établissements scolaires (Skolverket) qui remplaça, en 1991, la Direction générale de l'enseignement (Skolöverstyrelsen). À la même période, un nouveau discours sur la pratique éducative émergea, parallèlement à un nouveau système de formation des enseignants.

C'est dans le sillage de ces transformations de la politique scolaire que le gouvernement social-démocrate initia une réforme radicale de la formation des enseignants ${ }^{43}$. Comme nous allons le constater, cette réforme peut être considérée à bien des égards comme le point culminant d'un effort continu pour démocratiser le rôle des enseignants, et du même coup pour modifier la façon dont l'école s'oriente par rapport au temps ${ }^{44}$.

L'extrait suivant de la proposition révèle clairement sa continuité avec la politique précédente :

Le rôle de l'enseignant sera de plus en plus lié à la capacité de créer des rencontres personnelles. Les tâches professionnelles deviendront plus personnalisées au lieu d'être définies par des rôles. Plutôt que d'endosser un rôle, ou une tradition, chaque enseignant devra gagner et mériter sa place - et ainsi donc son autorité. L'autorité est quelque chose que l'on acquiert dans un processus démocratique. ${ }^{45}$

C'est en particulier l'idée que l'enseignant doit avoir la capacité de créer des " rencontres personnelles ", et par conséquent que ses tâches deviennent de plus en plus individuelles, qui nous intéresse ici $^{46}$. Cet appel aux enseignants doit être compris à la lumière du désir de former des professeurs prédisposés à s'adresser à chaque élève en tenant compte de ses expériences particulières. Nous voilà donc confrontés à une situation où l'autorité du professeur est considérée comme une question de négociation, où cette autorité doit être explicitement reconnue comme telle par l'élève. Afin de déléguer plus d'influence à l'élève, il est nécessaire que l'enseignant « considère que les élèves, en prenant en compte ce que l'on peut

43 Projet de loi pour une formation renouvelée des professeurs 1999-2000: 135.

44 Tomas Wedin, The Aporia of Equality. A Historico-Political Approach to Swedish Educational Politics 1946-2000, Göteborg, Göteborgs universitet, 2018.

45 Projet de loi 1999-2000: 135, p. 8. En revanche, l'expérience d'une perte d'autorité dans la salle de classe dont beaucoup de professeurs ont témoigné est un concept ancien, voir : Joakim Landahl, Auktoritet och ansvar. Lärares fostrans- och omsorgsarbete $i$ historisk belysning, Stockholm, Stockholms universitet, 2006, p. 130.

46 Projet de loi 1999-2000: 135, p. 8. 
raisonnablement leur demander, veuillent et puissent prendre plus de responsabilités dans leurs études et leur situation à l'école ", car cela est la meilleure manière de développer leurs attitudes démocratiques ${ }^{47}$.

Des idées similaires se retrouvent déjà dans une enquête de 1985 visant explicitement à fournir une base de réflexion pour donner plus d'influence aux élèves à l'école. Dans cette enquête - et c'est très révélateur pour ce qui nous intéresse -, la notion de responsabilité repose sur l'idée égalitaire selon laquelle tous les individus sont nés créatifs et compétents. Chaque élève doit donc exercer une plus grande influence qu'auparavant sur son destin ${ }^{48}$. Cet idéal d'égalité, ainsi lié aux capacités supposées de chacun, est censé favoriser l'interaction immédiate entre l'élève, avec ses expériences privées et sa situation particulière, et l'enseignant.

C'est un idéal qui va de pair avec l'idée d'une autorité négociée, et avec la volonté de créer une relation plus égale entre élève et professeur afin de démocratiser l'école. Dorénavant, le professeur doit " mettre en scène une activité impliquant que les élèves et les professeurs développent un respect mutuel ${ }^{49}$. De cette façon, les contours d'une situation d'apprentissage prennent forme là où l'enseignant œuvre avec les élèves, en créant alors une dynamique d'accès aux savoirs ${ }^{50}$. Ainsi, nous entrevoyons une conception des savoirs qui semble être fondée sur un acte performatif. Bien que la relativisation des savoirs soit ici intégrée au niveau discursif dans le nouveau langage managérial, la continuité par rapport à la conception de l'éducation et de l'égalité qui nous intéresse est indéniable. Contrairement à la conviction des premières décennies de l'après-guerre, où l'introduction à un monde dépassant l'individu était censée être nécessaire pour devenir citoyen (à travers le passé vers le futur), je soutiens que cela montre comment la préférence pour l'immédiateté a progressé au détriment des autres horizons temporels.

Un exemple est la discussion concernant le concept de «savoir » dans l'enquête SOU 1992 : 94 préparant les réformes au tournant des années 1990. Conformément à la nouvelle approche rendue explicite à travers la transformation en verbe du substantif suédois savoir (" savoirer», kunskapa), il n’y a ni bonne ni mauvaise

47 SOU 1992 : 94, p. 106.

48 Enquête avec le titre éloquent École pour la participation, SOU 1985 : 30, qui faisait partie d'une plus grande enquête intitulée Enquête sur la démocratie de 1983 [1983-års demokratiberedning].

49 Projet de loi $1999-2000: 135$, p. 8.

50 Ce que le chercheur néerlandais Gert Biesta décrit comme un déplacement de l'éducation à l'apprentissage (lärande) : Gert Biesta, Bortom lärandet, Lund, Studentlitteratur, 2012, p. 23. 
réponse, c'est "le travail qui est l'objectif " ${ }^{51}$. Selon l'apprentissage socioculturel, le savoir devrait être entendu comme :

[...] ni quelque chose d'extérieur ni d'intérieur, ni extérieur à l'individu ni contenu dans l'individu, mais plutôt quelque chose qui se trouve entre l'individu et l'environnement. Une partie importante de cet environnement est constituée par les tiers, le contexte social dans lequel le savoir est communiqué à travers le langage. ${ }^{52}$

C'est à la lumière de ces nouvelles idées autour du concept de savoir, condensées à travers l'idéal de l'apprentissage socioculturel, qu'il faut comprendre la référence du projet de loi pour une formation renouvelée des professeurs 1999-2000 dans la mise en scène par l'enseignant de « situations d'apprentissage ». Il est souligné dans ce même projet de loi que les élèves doivent participer à la planification des cours en collaborant avec les enseignants ; cela afin de " déterminer comment les objectifs de l'activité seront atteints " $"$. La tâche du nouvel enseignant devient ainsi, en premier lieu, de guider les élèves et de leur fournir les « conditions pour évaluer, examiner de façon critique et traiter l'information recueillie en savoirs utiles $"^{54}$.

\section{Conclusion}

Dans cet article, j'ai tenté de montrer comment les transformations du système éducatif suédois pendant la période de l'après-guerre pouvaient être comprises à la lumière de l'égalité en les abordant dans leurs trois dimensions temporelles. À travers le triangle élève/professeur/discipline, j'ai analysé comment un idéal d'égalité s'est développé tout au long de la période. Comme nous l'avons vu, nous pouvons discerner trois phases générales de ce processus : au départ, avec l'enquête de 1946, nous trouvons la tentative de réformer substantiellement l'école afin de la rendre plus démocratique et égalitaire. Il y est suggéré que les intérêts des élèves doivent jouer un rôle plus central dans l'enseignement. Cependant, cette enquête s'inscrit dans une conception qui inclut encore les trois dimensions temporelles. La finalité était d'éduquer les élèves pour devenir des citoyens égaux à travers un héritage commun.

51 Skola för bildning SOU 1992 : 94, p. 67. À comparer avec le raisonnement de Linderoth autour de l'épistémologie relativiste dans : Jonas Linderoth, Lärarens återkomst. Från förvirring till upprättelse, Stockholm, Natur \& kultur, 2016, p. 38ff.

52 Skola för bildning SOU 1992 : 94, p. 73. Voir aussi la façon dont l'influent pédagogue suédois Roger Säljö décrit l'apprentissage socioculturel dans un ancien livre de cours pour la formation des professeurs : "Selon la perspective socioculturelle, les savoirs ne sont pas quelque chose que l'individu possède, sous forme d'entités préemballées, emmagasinées dans une réserve ", Roger Säljö, Lärande i praktiken, Stockholm, Prisma, 2000, p. 125.

53 Projet de loi 1999-2000: 135, p. 10.

54 Ibid., p. 9. 
Dans un deuxième temps, dans les années 1970, une évolution cruciale a eu lieu dans la manière d'envisager le rôle du professeur et le contenu des disciplines. J'ai souligné comment cela fut en partie motivé par la conviction que - au nom de la démocratisation du système éducatif - l'autodétermination de l'élève devait exercer une influence plus importante sur son projet d'études. L'égalité est dorénavant censée être réalisée dans le système éducatif et dans le présent. Ensuite, dans la troisième phase, au cours des années 1990, nous avons vu comment l'idée d'une autorité négociée pourrait être comprise comme la continuation de cette tendance égalisatrice à confiner l'élève dans le présentisme ${ }^{55}$. L'injonction aux enseignants de personnaliser l'exercice de leur métier est emblématique de cette évolution. La réarticulation des tâches de l'enseignant témoigne ainsi d'un approfondissement de la transmutation de l'école comme forme institutionnelle évoquée dans cet article.

Dans une optique plus vaste, la tendance qui consiste à se concentrer de plus en plus sur l'élève là où il se trouve doit être comprise comme un déplacement du centre de gravité : du monde extérieur, médiatisé par un cadre institutionnel - ici représenté par l'école - vers l'individu, là où il se situe au moment présent ${ }^{56}$. Toutes les tendances mises en évidence, selon lesquelles l'enseignant doit laisser plus de place aux expériences privées des élèves (au détriment d'une transmission des savoirs), pourraient être considérées comme des manifestations de l'expansion d'un idéal d'égalité centré sur l'immédiateté et sur l'individu plutôt que sur la tradition ou l'avenir. Cette tendance peut être observée dans les documents des années 1970, où les enseignants sont sommés d'endosser un rôle de mentor, d'apprendre à apprendre et à envisager leur rôle à travers une autorité négociée. Et c'est pour cela que je constate un glissement vers des formes de relation calquées sur les idéaux de la sphère privée : ce qui est mis en avant, c'est moins le rôle institutionnel de l'enseignant que sa personnalité ; l'importance accrue des expériences particulières des élèves milite dans le même sens.

Le point de vue dominant, qui souligne une rupture dans la politique éducative autour des années 1990, a justement mis en avant l'aspect libéral des réformes de cette époque. Le discours explicitement libéral, accentuant la liberté de choix pour chaque élève, est la meilleure illustration de cette rupture tant au niveau du discours que des politiques. Néanmoins, je soutiens que les initiatives visant à introduire des méthodes plus démocratiques dans l'école ont paradoxalement

55 Un aspect des changements que je n'ai pas abordé dans cet article, mais que Lundgren souligne dans l'entretien publié dans ce numéro, est comment la transmutation de l'économie a aussi soutenu cette gravitation vers le présent. Le rapport entre ces deux causes est abordé dans : Tomas Wedin, The Aporia, op. cit.

56 Pour un aperçu plus précis de l'approche institutionnelle, voir : Anton Zijderveld, The Institutional Imperative, Amsterdam, Amsterdam University Press, 2000. 
sapé - avec des motivations progressistes - les fondations d'un système éducatif bâti sur un ordre collectif. Et ainsi, peut-être à leur insu, elles ont progressivement contribué à l'imbrication plus forte entre des formes d'interaction propres à la sphère privée, d'une part, et à l'école publique, d'autre part - et ce au nom d'une conception originale de l'égalité démocratique.

Plus largement, il semble que nous soyons confrontés à une contradiction plus profonde au cœur même de l'ordre institutionnel sur lequel la modernité est fondée. D'un côté, nos sociétés s'appuient sur une idéologie progressiste, particulièrement forte en Suède, pour laquelle " l'adversaire dialectique est, à chaque reprise, la force inerte de l'habitude $"^{57}$. D'un autre côté, l'idée même de la société est redevable d'un cadre institutionnel stable, sans lequel aucune pensée du progrès social ne saurait émerger. De ce point de vue, l'exemple des transformations de l'éducation suédoise pendant la période de l'après-guerre semble n'être qu'une illustration d'un paradoxe plus général au cœur du projet moderne dans son expression contemporaine.

57 Piero Colla, L'héritage impossible, op. cit., p. 853. 